\title{
Resources Investigation of Indigenous Plants in Nanchang and Their Application in Urban Landscape
}

\author{
Xu Qin ${ }^{1}$ \\ ${ }^{1}$ Landscape Architecture Department, Nanchang Institute of Technology, Nanchang, Jiangxi, China
}

\begin{abstract}
To improve bio-diversity in cities, we need to focus on indigenous plants in greening initiatives. In this study, the indigenous plants in Nanchang were investigated to analyze the current situations of indigenous plants in this region and their application in urban greening in Nanchang. The problems in using indigenous plants for greening in Nanchang City were analyzed and corresponding suggestions were made.
\end{abstract}

\section{Introduction}

Biodiversity is an essential condition for human survival, a basis of sustainable socio-economic development, and the guarantee of ecological safety and food security. Currently, nations across the world have been taking measures to address the crisis of global biodiversity. The biodiversity in cities is closely connected to plant diversity, and hence plant landscaping should rely mainly on indigenous plants, with introduced plants as supplements. Indigenous plants refer to the plant species native to the local region ${ }^{[1]}$, featuring high adaptability, strong resilience, and convenient maintenance; they often do no harm to the local ecosystem and are likely to form regional landscapes. Many cities in China present identikit landscapes, as they introduce ornamental plants from other places and overlook the indigenous ones, which leads to a shortage of local specialties and reduces biodiversity. Nanchang boasts a rich range of indigenous plants, and landscape designers have encouraged use of these resources. However, heavy dependence on introduced species is still pronounced because the urban planners often pursue novelty, excellence, rarity and peculiarity in planning, but give short the indigenous plants short shrift. Thus, to understand the distribution and fully utilize indigenous plants is of great value for protecting the ecological well-being and biodiversity in cities.

\section{Study area and research methods}

\subsection{Natural conditions of the study area}

Nanchang $\left(115^{\circ} 27^{\prime}-116^{\circ} 35^{\prime} \mathrm{E}, 28^{\circ} 10^{\prime}-29^{\circ} 11^{\prime} \mathrm{N}\right)$ is located in mid-north of Jiangxi Province, in the lower reaches of Ganjiang River and Fuhe River, and on the southwestern bank of Poyang Lake. It is adjacent to Yugan and Dongxiang to the east, Linchuan and Fengcheng to the south, Gao'an, Fengxin and Jing'an to the west, and Yongxiu, Duchang, and Poyang to the north. Its maximum south-north stretch is $121 \mathrm{~km}$, and the maximum east-west stretch is $108 \mathrm{~km}$, with the Xiyaowu Hill in Xiyao Lake at the peak of Dianmei Mountain marking its highest altitude of $841.4 \mathrm{~m}$. The area is dominated by Poyang Lake Plain, with mountains, hills, ridges and plains interwoven into the local geological fabric. The eastern and southern areas are flat, while the western and northern parts are dominated by hills. The region is watered densely by rivers and dotted by lakes and ponds. Nanchang is subject to the subtropical monsoon climate, featured by humidity and mild temperatures, sufficient sunlight, longer summers and winters but shorter springs and autumns. The average annual temperature is within a range from $17^{\circ} \mathrm{C}$ to $17.7^{\circ} \mathrm{C}$, with the highest temperature in history reaching $40.9^{\circ} \mathrm{C}$, and the historically low temperature at $-15.2^{\circ} \mathrm{C}$. Situated in the subtropical area in the northern hemisphere, Nanchang is subject to the impact of East Asian monsoon, thus features a subtropical monsoon climate. In winters, the northerly wind dominates, and in summers the southerly wind prevails. The annual precipitation reaches $1600-1700 \mathrm{~mm}$, with $147-157$ raining days; the average days with rainstorms per year are 5.6 days, and the annual average relative humidity is $78.5 \%$. The annual sunshine duration is $1723-1820$ hours, and the average yearly percentage of sunshine is $40 \%$, with June and August witnessing the largest percentage, while February and March marking the lowest percentage. The annual frost-free days are $251-272$ days, which is favorable for growth of plants.

\subsection{Investigation methods}

Path investigations and key investigations were combined in this study to investigate the inside areas and the peripheries of Nanchang City. The peripheries involve the Meiling Forest Park, Shengshuitang Forest Park, and Xiangshan Forest Park. Inside the city, the investigated areas include large parks and gardens like 
Yaohu Lake Forest Botanical Garden, Aixi Lake Wetland Park, Bayi Park, People's Park, residential communities, organizations and roads with good greening effects. The investigation items include the types, features, biotope and the ornamental value of the plants. Fieldwork was conducted and statistical analysis was made, together with photo-shooting, recording and archiving.

\section{Investigation results}

\subsection{Plant resources of Nanchang}

In China, Nanchang is located in the northern part of the mid-Asian subtropical evergreen broad-leaved forest area, at the conjunction of the castanopsis, nanmu, and schima superba forests of mountains and hills in Hunan and Jiangxi Province, the castanopsis, nanmu, schima superba, and coniferopsida forests in the mountains and hills of Jiuling Mountain of the cultural vegetation region, the castanopsis, nanmu, and coniferopsida forests of the mid- and upper-reach of Jinjiang River and Yuanshui River, the castanopsis, nanmu and coniferopsida sub-forests of the lower reaches of Ganjiang River, Fuhe River, and Xinjiang River. The dominating plants include pines, cedars and camphor trees, the gingko renowned as "living fossils", metasequoia, as well as trees of Actinidia chinensis that is known as the "king of fruits".

Fieldwork investigation results and statistical analysis reveal that Nanchang boasts 1708 higher plants, dominated by timber woods and fuelwood forests. The major families of plants include species of the Fagaceae, Lauraceae, Rosaceae, theaceae, hamamelidaceae, Elaeocarpaceae, Euphorbiaceae, Aquifoliaceae, symplocaceae, and Poaceae.

\subsection{Overview of the types of indigenous plants in Nanchang}

According to the Flora of Jiangxi ${ }^{[2]-[4]}$, the investigation results revealed that the indigenous plants in Nanchang include 129 families, 317 genera and 451 species, among which there are 14 families, 16 genera and 17 species of pteridophyte, 3 families, 3 genera and 3 species of gymnosperm, 112 families, 298 genera and 431 species of angiosperm, as shown in Table 1.

Statistical analysis shows that the greening plants in Nanchang belong to 125 families, 385 genera and 577 species. The indigenous greening plants in Nanchang include 58 families, 87 genera and 96 species of plants, 3 families, 3 genera and 3 species of gymnosperm, 52 families, 83 genera and 92 species of angiosperm. Among these categories, there are 6 families, 6 genera and 6 species of evergreen arbor trees, 10 families, 11 genera and 11 species of deciduous arbor trees, 6 families, 7 genera and 8 species of evergreen deciduous shrubs, 2 families, 2 genera and 2 species of evergreen vines, 4 families, 5 genera and 5 species of deciduous vines, as shown in Table 2.
Table 1. The components of families, genera and species of indigenous plants in Nanchang

\begin{tabular}{|c|c|c|}
\hline Family & Genus & Species \\
\hline Lycopodiaceae & 1 & 1 \\
\hline Selaginellaceae & 1 & 1 \\
\hline Equisetaceae & 1 & 1 \\
\hline Osmundaceae & 1 & 1 \\
\hline Gleicheniaceae & 1 & 1 \\
\hline Lygodiaceae & 1 & 1 \\
\hline Lindsaeaceae & 1 & 1 \\
\hline Hypolepidaceae & 1 & 1 \\
\hline Hemionitidaceae & 1 & 1 \\
\hline Pteridaceae & 2 & 2 \\
\hline Pteridiaceae & 1 & 1 \\
\hline Athyriaceae & 1 & 1 \\
\hline Blechnaceae & 1 & 1 \\
\hline Dryopteridaceae & 2 & 3 \\
\hline Pinaceae & 1 & 1 \\
\hline Taxodiaceae & 1 & 1 \\
\hline Cephalotaxaceae & 1 & 1 \\
\hline Dicotyledoneae & 1 & 1 \\
\hline Schisandraceae & 2 & 3 \\
\hline Lauraceae & 4 & 8 \\
\hline Ranunculaceae & 6 & 11 \\
\hline Ceratophyllaceae & 1 & 1 \\
\hline Berberidaceae & 2 & 2 \\
\hline Lardizabalaceae & 2 & 2 \\
\hline Menispermaceae & 2 & 2 \\
\hline Aristolochiaceae & 1 & 2 \\
\hline Saururaceae & 1 & 1 \\
\hline Chloranthaceae & 2 & 2 \\
\hline Papaveraceae & 1 & 1 \\
\hline Fumariaceae & 1 & 2 \\
\hline Cruciferae & 2 & 4 \\
\hline Violaceae & 1 & 4 \\
\hline Polygalaceae & 1 & 1 \\
\hline Crassulaceae & 1 & 3 \\
\hline Caryophyllaceae & 3 & 3 \\
\hline Molluginaceae & 1 & 1 \\
\hline Portulacaceae & 1 & 1 \\
\hline Polygonaceae & 3 & 7 \\
\hline Amaranthaceae & 2 & 2 \\
\hline Geraniaceae & 1 & 1 \\
\hline Oxalidaceae & 1 & 1 \\
\hline Lythraceae & 1 & 1 \\
\hline Onagraceae & 1 & 1 \\
\hline Thymelaeaceae & 1 & 1 \\
\hline Pittosporaceae & 1 & 1 \\
\hline Cucurbitaceae & 2 & 2 \\
\hline Theaceae & 6 & 11 \\
\hline Actinidiaceae & 1 & 1 \\
\hline Myrtaceae & 1 & 1 \\
\hline Melastomataceae & 3 & 3 \\
\hline Hypericaceae & 1 & 3 \\
\hline Tiliaceae & 2 & 2 \\
\hline Elaeocarpaceae & 1 & 2 \\
\hline Malvaceae & 2 & 2 \\
\hline Euphorbiaceae & 9 & 16 \\
\hline Daphniphyllaceae & 1 & 1 \\
\hline Iteaceae & 1 & 1 \\
\hline Hydrangeaceae & 2 & 3 \\
\hline Rosaceae & 8 & 18 \\
\hline Mimosaceae & 2 & 2 \\
\hline Caesalpiniaceae & 1 & 2 \\
\hline Fabaceae & 10 & 15 \\
\hline Stachyuraceae & 1 & 1 \\
\hline Hamamelidaceae & 4 & 4 \\
\hline Buxaceae & 1 & 1 \\
\hline Salicaceae & 1 & 1 \\
\hline Myricaceae & 1 & 1 \\
\hline Betulaceae & 1 & 1 \\
\hline Corylaceae & 1 & 1 \\
\hline Fagaceae & 5 & 9 \\
\hline Ulmaceae & 3 & 3 \\
\hline Moraceae & 3 & 8 \\
\hline Urticaceae & 7 & 12 \\
\hline
\end{tabular}




\begin{tabular}{|c|c|c|}
\hline Family & Genus & Species \\
\hline Cannabaceae & 1 & 1 \\
\hline Aquifoliaceae & 1 & 6 \\
\hline Celastraceae & 1 & 2 \\
\hline Rhamnaceae & 2 & 2 \\
\hline Vitaceae & 3 & 5 \\
\hline Rutaceae & 2 & 3 \\
\hline Simaroubaceae & 2 & 2 \\
\hline Meliaceae & 1 & 1 \\
\hline Aceraceae & 1 & 4 \\
\hline Sabiaceae & 2 & 3 \\
\hline Staphyleaceae & 2 & 2 \\
\hline Anacardiaceae & 2 & 3 \\
\hline Juglandaceae & 2 & 2 \\
\hline Alangiaceae & 1 & 1 \\
\hline Araliaceae & 6 & 7 \\
\hline Umbelliferae & 5 & 7 \\
\hline Ericaceae & 1 & 4 \\
\hline Ebenaceae & 1 & 1 \\
\hline Myrsinaceae & 3 & 4 \\
\hline Styracaceae & 2 & 4 \\
\hline Symplocaceae & 1 & 6 \\
\hline Strychnaceae & 1 & 2 \\
\hline Oleaceae & 2 & 4 \\
\hline Apocynaceae & 1 & 1 \\
\hline Asclepiadaceae & 1 & 1 \\
\hline Rubiaceae & 11 & 15 \\
\hline Caprifoliaceae & 4 & 7 \\
\hline Valerianaceae & 1 & 2 \\
\hline Compositae & 23 & 27 \\
\hline Primulaceae & 1 & 2 \\
\hline Plantaginaceae & 1 & 1 \\
\hline Campanulaceae & 2 & 2 \\
\hline Lobeliaceae & 1 & 3 \\
\hline Boraginaceae & 2 & 2 \\
\hline Solanaceae & 1 & 2 \\
\hline Convolvulaceae & 2 & 2 \\
\hline Cuscutaceae & 1 & 1 \\
\hline Scrophulariaceae & 6 & 6 \\
\hline Acanthaceae & 2 & 2 \\
\hline Verbenaceae & 5 & 8 \\
\hline Labiatae & 10 & 13 \\
\hline Hydrocharitaceae & 1 & 1 \\
\hline Potamogetonaceae & 1 & 1 \\
\hline Commelinaceae & 1 & 1 \\
\hline Zingiberaceae & 1 & 1 \\
\hline Liliaceae & 7 & 8 \\
\hline Smilacaceae & 2 & 3 \\
\hline Araceae & 4 & 4 \\
\hline Lemnaceae & 2 & 2 \\
\hline Typhaceae & 1 & 1 \\
\hline Iridaceae & 1 & \\
\hline Stemonaceae & 1 & 1 \\
\hline Dioscoreaceae & 1 & 1 \\
\hline Juncaceae & 1 & 2 \\
\hline Cyperaceae & 7 & 12 \\
\hline Gramineae & 19 & 24 \\
\hline
\end{tabular}

Table 2. Statistical analysis results of indigenous plants in urban landscape in Nanchang

\begin{tabular}{|c|c|c|c|c|}
\hline Plant name & Family & Genus & Latin name & Category \\
\hline $\begin{array}{l}\text { Masson } \\
\text { pine }\end{array}$ & Qinaceae & Pinus & $\begin{array}{l}\text { Pinus } \\
\text { massoniana }\end{array}$ & $\begin{array}{l}\text { Evergreen } \\
\text { tree }\end{array}$ \\
\hline Fir & Taxodiaceae & $\begin{array}{l}\text { Cunningha- } \\
\text { mia }\end{array}$ & $\begin{array}{l}\text { Cunninghamia } \\
\text { lanceolata }\end{array}$ & $\begin{array}{l}\text { Evergreen } \\
\text { tree }\end{array}$ \\
\hline $\begin{array}{l}\text { Cinnamom- } \\
\text { um } \\
\text { camphora }\end{array}$ & Lauraceae & $\begin{array}{l}\text { Cinnamom- } \\
\text { um }\end{array}$ & $\begin{array}{l}\text { Cinnamomum } \\
\text { camphora }\end{array}$ & $\begin{array}{l}\text { Evergreen } \\
\text { tree }\end{array}$ \\
\hline $\begin{array}{l}\text { Sassafras } \\
\text { tzumu }\end{array}$ & Lauraceae & Sassafras & $\begin{array}{l}\text { Sassafras } \\
\text { tzumu }\end{array}$ & $\begin{array}{l}\text { Deciduous } \\
\text { tree }\end{array}$ \\
\hline $\begin{array}{l}\text { Muskroot-li } \\
\text { ke } \\
\text { semiaquile } \\
\text { g-ia herb }\end{array}$ & Ranunculaceae & $\begin{array}{l}\text { Semiaquile- } \\
\text { gia }\end{array}$ & $\begin{array}{l}\text { Semiaquilegia } \\
\text { adoxoides }\end{array}$ & $\begin{array}{l}\text { Perennial } \\
\text { herb }\end{array}$ \\
\hline $\begin{array}{l}\text { Nandina } \\
\text { domestica }\end{array}$ & Berberidaceae & Nandina & $\begin{array}{l}\text { Nandina } \\
\text { domestica }\end{array}$ & $\begin{array}{l}\text { Evergreen } \\
\text { shrub }\end{array}$ \\
\hline $\begin{array}{l}\text { Japanese } \\
\text { Snailseed } \\
\text { Root }\end{array}$ & $\begin{array}{l}\text { Menispermacea } \\
e\end{array}$ & Cocculus & $\begin{array}{l}\text { Cocculus } \\
\text { orbiculatus }\end{array}$ & $\begin{array}{l}\text { Deciduous } \\
\text { vine }\end{array}$ \\
\hline
\end{tabular}

\begin{tabular}{|c|c|c|c|c|}
\hline Plant name & Family & Genus & Latin name & Category \\
\hline $\begin{array}{l}\text { Houttuynia } \\
\text { cordata }\end{array}$ & Saururaceae & Houttuynia & $\begin{array}{l}\text { Houttuynia } \\
\text { cordata }\end{array}$ & $\begin{array}{l}\text { Perennial } \\
\text { herb }\end{array}$ \\
\hline $\begin{array}{l}\text { Indian } \\
\text { rorippa } \\
\text { herb }\end{array}$ & Eutrema & Rorippa & Rorippa indica & $\begin{array}{l}\text { Annual } \\
\text { herb }\end{array}$ \\
\hline $\begin{array}{l}\text { Chinese } \\
\text { violet }\end{array}$ & Violaceae & Viola & Viola philippica & $\begin{array}{l}\text { Perennial } \\
\text { herb }\end{array}$ \\
\hline $\begin{array}{l}\text { Japonese } \\
\text { pearlwort } \\
\text { herb }\end{array}$ & $\begin{array}{l}\text { Caryophyllacea } \\
e\end{array}$ & Sagina & $\begin{array}{l}\text { Sagina } \\
\text { japonica }\end{array}$ & $\begin{array}{l}\text { Annual } \\
\text { herb }\end{array}$ \\
\hline Chickweed & $\begin{array}{l}\text { Caryophyllacea } \\
e\end{array}$ & Stellaria & Stellaria media & $\begin{array}{l}\text { Annual } \\
\text { herb }\end{array}$ \\
\hline $\begin{array}{l}\text { Persicaria } \\
\text { hydropiper }\end{array}$ & Polygonacae & Polygonum & $\begin{array}{l}\text { Polygonum } \\
\text { hydropiper }\end{array}$ & $\begin{array}{l}\text { Annual } \\
\text { herb }\end{array}$ \\
\hline $\begin{array}{l}\text { Creeping } \\
\text { oxalis }\end{array}$ & Oxalidaceae & Oxalis & $\begin{array}{l}\text { Oxalis } \\
\text { corniculata }\end{array}$ & $\begin{array}{l}\text { Perennial } \\
\text { herb }\end{array}$ \\
\hline Sasanqua & Theaceae & Camellia & $\begin{array}{l}\text { Camellia } \\
\text { oleifera }\end{array}$ & $\begin{array}{l}\text { Evergreen } \\
\text { shrub }\end{array}$ \\
\hline Tea tree & Theaceae & Camellia & $\begin{array}{l}\text { Camellia } \\
\text { sinensis }\end{array}$ & $\begin{array}{l}\text { Evergreen } \\
\text { shrub }\end{array}$ \\
\hline $\begin{array}{l}\text { Gurgeon } \\
\text { stopper }\end{array}$ & Myrtaceae & Syzygium & $\begin{array}{l}\text { Syzygium } \\
\text { buxifolium }\end{array}$ & $\begin{array}{l}\text { Evergreen } \\
\text { shrub }\end{array}$ \\
\hline $\begin{array}{l}\text { Hypericum } \\
\text { monogynu- } \\
\mathrm{m}\end{array}$ & Hypericaceae & Hypericum & $\begin{array}{l}\text { Hypericum } \\
\text { monogynum }\end{array}$ & $\begin{array}{l}\text { Evergreen } \\
\text { shrub }\end{array}$ \\
\hline $\begin{array}{l}\text { Elaeocarpu } \\
\text {-s } \\
\text { glabripetalu } \\
- \text { s }\end{array}$ & $\begin{array}{l}\text { Elaeocarpacea } \\
e\end{array}$ & $\begin{array}{l}\text { Elaeocarpu } \\
-s\end{array}$ & $\begin{array}{l}\text { Elaeocarpus } \\
\text { glabripetalus }\end{array}$ & $\begin{array}{l}\text { Evergreen } \\
\text { tree }\end{array}$ \\
\hline $\begin{array}{l}\text { Acalypha } \\
\text { brachystach } \\
\text {-ya }\end{array}$ & Euphorbiaceae & Acalypha. & $\begin{array}{l}\text { Acalypha } \\
\text { supera }\end{array}$ & $\begin{array}{l}\text { Annual } \\
\text { herb }\end{array}$ \\
\hline $\begin{array}{l}\text { Humifuse } \\
\text { euphorbia } \\
\text { herb }\end{array}$ & Euphorbiaceae & Euphorbia & $\begin{array}{l}\text { Euphorbia } \\
\text { humifusa }\end{array}$ & $\begin{array}{l}\text { Annual } \\
\text { herb }\end{array}$ \\
\hline $\begin{array}{l}\text { Under-leaf } \\
\text { pearl }\end{array}$ & Euphorbiaceae & Phyllanthus & $\begin{array}{l}\text { Phyllanthus } \\
\text { urinaria }\end{array}$ & $\begin{array}{l}\text { Annual } \\
\text { herb }\end{array}$ \\
\hline $\begin{array}{l}\text { Sapium } \\
\text { sebiferum }\end{array}$ & Euphorbiaceae & Sapium & $\begin{array}{l}\text { Sapium } \\
\text { sebiferum }\end{array}$ & $\begin{array}{l}\text { Deciduous } \\
\text { tree }\end{array}$ \\
\hline $\begin{array}{l}\text { Mock- } \\
\text { strawberry }\end{array}$ & Rosaceae & Duchesnea & $\begin{array}{l}\text { Duchesnea } \\
\text { indica }\end{array}$ & $\begin{array}{l}\text { Perennial } \\
\text { herb }\end{array}$ \\
\hline $\begin{array}{l}\text { Freyn } \\
\text { cinquefoil } \\
\text { herb }\end{array}$ & Rosaceae & Potentilla & $\begin{array}{l}\text { Potentilla } \\
\text { freyniana }\end{array}$ & $\begin{array}{l}\text { Perennial } \\
\text { herb }\end{array}$ \\
\hline $\begin{array}{l}\text { Pyracantha } \\
\text { crenatoserr } \\
\text { a-ta }\end{array}$ & Rosaceae & Pyracantha & $\begin{array}{l}\text { Pyracantha } \\
\text { fortuneana }\end{array}$ & $\begin{array}{l}\text { Evergreen } \\
\text { shrub }\end{array}$ \\
\hline $\begin{array}{l}\text { Rubus } \\
\text { althaeoides }\end{array}$ & Rosaceae & Rubus & $\begin{array}{l}\text { Rubus } \\
\text { corchorifolius }\end{array}$ & $\begin{array}{l}\text { Deciduous } \\
\text { shrub }\end{array}$ \\
\hline $\begin{array}{l}\text { Rubus } \\
\text { rosaefolius }\end{array}$ & Rosaceae & Rubus & $\begin{array}{l}\text { Rubus } \\
\text { rosifolius }\end{array}$ & $\begin{array}{l}\text { Deciduous } \\
\text { shrub }\end{array}$ \\
\hline $\begin{array}{l}\text { Mimosa } \\
\text { pudica }\end{array}$ & Mimosaceae & Mimosa & Mimosa pudica & $\begin{array}{l}\text { Perennial } \\
\text { herb }\end{array}$ \\
\hline $\begin{array}{l}\text { Japanese } \\
\text { clover herb }\end{array}$ & Papillionaceae & $\begin{array}{l}\text { Kummero- } \\
\text { wia }\end{array}$ & $\begin{array}{l}\text { Kummerowia } \\
\text { striata }\end{array}$ & $\begin{array}{l}\text { Annual } \\
\text { herb }\end{array}$ \\
\hline $\begin{array}{l}\text { Pueraria } \\
\text { lobata }\end{array}$ & Papillionaceae & Pueraria & $\begin{array}{l}\text { Pueraria } \\
\text { motana var. } \\
\text { lobata }\end{array}$ & $\begin{array}{l}\text { Deciduous } \\
\text { vine }\end{array}$ \\
\hline $\begin{array}{l}\text { Liquidamb- } \\
\text { ar } \\
\text { Styraciflua }\end{array}$ & $\begin{array}{l}\text { Hamamelidace } \\
\text { ae }\end{array}$ & $\begin{array}{l}\text { Liquidamb- } \\
\text { ar }\end{array}$ & $\begin{array}{l}\text { Liquidambar } \\
\text { formosana }\end{array}$ & $\begin{array}{l}\text { Deciduous } \\
\text { tree }\end{array}$ \\
\hline $\begin{array}{l}\text { Loropetalu- } \\
\text { m chinense }\end{array}$ & $\begin{array}{l}\text { Hamamelidace } \\
\text { ae }\end{array}$ & $\begin{array}{l}\text { Loropetalu- } \\
m\end{array}$ & $\begin{array}{l}\text { Loropetalum } \\
\text { chinense }\end{array}$ & $\begin{array}{l}\text { Deciduous } \\
\text { shrub }\end{array}$ \\
\hline $\begin{array}{l}\text { Chinese } \\
\text { waxmyrtle }\end{array}$ & Myricaceae & Myrica & Myrica rubra & $\begin{array}{l}\text { Evergreen } \\
\text { tree }\end{array}$ \\
\hline $\begin{array}{l}\text { Quercus } \\
\text { alba }\end{array}$ & Fagaceae & Quercus & Quercus fabri & $\begin{array}{l}\text { Deciduous } \\
\text { tree }\end{array}$ \\
\hline $\begin{array}{l}\text { Chinese } \\
\text { hackberry } \\
\text { root-bark }\end{array}$ & Ulmaceae & Celtis & Celtis sinensis & $\begin{array}{l}\text { Deciduous } \\
\text { tree }\end{array}$ \\
\hline $\begin{array}{l}\text { Chinese } \\
\text {-elm }\end{array}$ & Ulmaceae & Ulmus & $\begin{array}{l}\text { Ulmus } \\
\text { parvifolia }\end{array}$ & $\begin{array}{l}\text { Deciduous } \\
\text { tree }\end{array}$ \\
\hline $\begin{array}{l}\text { Common } \\
\text { papermulbe } \\
\text {-rry } \\
\text { root-bark }\end{array}$ & Moraceae & $\begin{array}{l}\text { Broussoneti } \\
-a\end{array}$ & $\begin{array}{l}\text { Broussonetia } \\
\text { papyrifera }\end{array}$ & $\begin{array}{l}\text { Deciduous } \\
\text { tree }\end{array}$ \\
\hline $\begin{array}{l}\text { Boehmeria } \\
\text { clidemioide } \\
\text {-s var } \\
\text { diffusa }\end{array}$ & Urticaeae & Boehmeria & $\begin{array}{l}\text { Boehmeria } \\
\text { clidemioides } \\
\text { var. diffusa }\end{array}$ & $\begin{array}{l}\text { Perennial } \\
\text { herb }\end{array}$ \\
\hline Ramie & Urticaeae & Boehmeria & $\begin{array}{l}\text { Boehmeria } \\
\text { nivea }\end{array}$ & $\begin{array}{l}\text { Deciduous } \\
\text { shrub }\end{array}$ \\
\hline $\begin{array}{l}\text { Suspended } \\
\text { leaf ramie }\end{array}$ & Urticaeae & Boehmeria & $\begin{array}{l}\text { Boehmeria } \\
\text { tricuspis }\end{array}$ & $\begin{array}{l}\text { Perennial } \\
\text { herb }\end{array}$ \\
\hline $\begin{array}{l}\text { Hirsute } \\
\text { gonostegia } \\
\text { herb }\end{array}$ & Urticaeae & Gonostegia & $\begin{array}{l}\text { Gonostegia } \\
\text { hirta }\end{array}$ & $\begin{array}{l}\text { Perennial } \\
\text { herb }\end{array}$ \\
\hline $\begin{array}{l}\text { Pilose } \\
\text { nanocnide } \\
\text { herb }\end{array}$ & Urticaeae & Nanocnide & $\begin{array}{l}\text { Nanocnide } \\
\text { lobata }\end{array}$ & $\begin{array}{l}\text { Perennial } \\
\text { herb }\end{array}$ \\
\hline $\begin{array}{l}\text { Japanese } \\
\text { cayratia }\end{array}$ & Vitaceae & Cayratia & $\begin{array}{l}\text { Cayratia } \\
\text { japonica }\end{array}$ & $\begin{array}{l}\text { Deciduous } \\
\text { vine }\end{array}$ \\
\hline
\end{tabular}




\begin{tabular}{|c|c|c|c|c|}
\hline Plant name & Family & Genus & Latin name & Category \\
\hline \\
\hline $\begin{array}{l}\text { Parthenocis } \\
\text {-sus } \\
\text { tricuspidata }\end{array}$ & Vitaceae & $\begin{array}{l}\text { Parthenoci } \\
\text { s-sus }\end{array}$ & $\begin{array}{l}\text { Parthenocissus } \\
\text { tricuspidata }\end{array}$ & $\begin{array}{l}\text { Deciduous } \\
\text { vine }\end{array}$ \\
\hline Ailanthus & Simarubaceae & Ailanthus & $\begin{array}{l}\text { Ailanthus } \\
\text { altissima }\end{array}$ & $\begin{array}{l}\text { Deciduous } \\
\text { tree }\end{array}$ \\
\hline $\begin{array}{l}\text { Chinaberry } \\
\text { tree fruit }\end{array}$ & Meliaceae & Melia & $\begin{array}{l}\text { Melia } \\
\text { azedarach }\end{array}$ & $\begin{array}{l}\text { Deciduous } \\
\text { tree }\end{array}$ \\
\hline Acer fabri & Aceraceae & Acer & Acer fabri & $\begin{array}{l}\text { Evergreen } \\
\text { tree }\end{array}$ \\
\hline $\begin{array}{l}\text { Rhus } \\
\text { semialata }\end{array}$ & Anacardiaceae & Rhus & Rhus chinensis & $\begin{array}{l}\text { Deciduous } \\
\text { shrub }\end{array}$ \\
\hline $\begin{array}{l}\text { Chinese } \\
\text { wingnut }\end{array}$ & Juglandaceae & Pterocarya & $\begin{array}{l}\text { Pterocarya } \\
\text { stenoptera }\end{array}$ & $\begin{array}{l}\text { Deciduous } \\
\text { tree }\end{array}$ \\
\hline Ivy & Araliaceae & Hedera & $\begin{array}{l}\text { Hedera } \\
\text { nepalensis var. } \\
\text { sinensis }\end{array}$ & $\begin{array}{l}\text { Evergreen } \\
\text { vine }\end{array}$ \\
\hline $\begin{array}{l}\text { Asiatic } \\
\text { pennywort } \\
\text { herb }\end{array}$ & Umbelliferae & Centella & $\begin{array}{l}\text { Centella } \\
\text { asiatica }\end{array}$ & $\begin{array}{l}\text { Perennial } \\
\text { herb }\end{array}$ \\
\hline $\begin{array}{l}\text { Nepal } \\
\text { pennywort } \\
\text { herb }\end{array}$ & Umbelliferae & $\begin{array}{l}\text { Hydrocotyl- } \\
e\end{array}$ & $\begin{array}{l}\text { Hydrocotyle } \\
\text { nepalensis }\end{array}$ & $\begin{array}{l}\text { Perennial } \\
\text { herb }\end{array}$ \\
\hline $\begin{array}{l}\text { Lawn } \\
\text { pennywort } \\
\text { herb }\end{array}$ & Umbelliferae & $\begin{array}{l}\text { Hydrocotyl- } \\
e\end{array}$ & $\begin{array}{l}\text { Hydrocotyle } \\
\text { sibthorpioides }\end{array}$ & $\begin{array}{l}\text { Perennial } \\
\text { herb }\end{array}$ \\
\hline $\begin{array}{l}\text { Common } \\
\text { hedgeparsle } \\
\text {-y fruit }\end{array}$ & Umbelliferae & Torilis & Torilis scabra & $\begin{array}{l}\text { Annual } \\
\text { herb }\end{array}$ \\
\hline $\begin{array}{l}\text { Ligustrum } \\
\text { quihouicarr. }\end{array}$ & Oleaceae & Ligustrum & $\begin{array}{l}\text { Ligustrum } \\
\text { quihoui }\end{array}$ & $\begin{array}{l}\text { Deciduous } \\
\text { shrub }\end{array}$ \\
\hline Retinervus & Apocynaceae & $\begin{array}{l}\text { Trachelosp } \\
\text { e-rmum }\end{array}$ & $\begin{array}{l}\text { Trachelosperm- } \\
\text { um jasminoides }\end{array}$ & $\begin{array}{l}\text { Evergreen } \\
\text { vine }\end{array}$ \\
\hline $\begin{array}{l}\text { Cape } \\
\text { jasmine }\end{array}$ & Rubiaceae & Gardenia & $\begin{array}{l}\text { Gardenia } \\
\text { jasminoides }\end{array}$ & $\begin{array}{l}\text { Evergreen } \\
\text { shrub }\end{array}$ \\
\hline $\begin{array}{l}\text { Oldenlandi- } \\
\text { a } \\
\text { chrysotrich } \\
\text { a }\end{array}$ & Rubiaceae & Hedyotis & $\begin{array}{l}\text { Hedyotis } \\
\text { chrysotricha }\end{array}$ & $\begin{array}{l}\text { Perennial } \\
\text { herb }\end{array}$ \\
\hline $\begin{array}{l}\text { Paederia } \\
\text { scandens } \\
\text { var. } \\
\text { tomentosa }\end{array}$ & Rubiaceae & Paederia & $\begin{array}{l}\text { Paederia } \\
\text { scandens var. } \\
\text { tomentosa }\end{array}$ & $\begin{array}{l}\text { Deciduous } \\
\text { vine }\end{array}$ \\
\hline $\begin{array}{l}\text { June snow } \\
\text { herb }\end{array}$ & Rubiaceae & Serissa & $\begin{array}{l}\text { Serissa } \\
\text { japonica }\end{array}$ & $\begin{array}{l}\text { Evergreen } \\
\text { shrub }\end{array}$ \\
\hline $\begin{array}{l}\text { Chinese } \\
\text { abelia }\end{array}$ & Caprifoliaceae & Abelia & $\begin{array}{l}\text { Abelia } \\
\text { chinensis }\end{array}$ & $\begin{array}{l}\text { Deciduous } \\
\text { shrub }\end{array}$ \\
\hline $\begin{array}{l}\text { Aster } \\
\text { tataricus }\end{array}$ & Compositae & Aster & $\begin{array}{l}\text { Aster } \\
\text { ageratoides }\end{array}$ & $\begin{array}{l}\text { Perennial } \\
\text { herb }\end{array}$ \\
\hline $\begin{array}{l}\text { Horseweed } \\
\text { herb }\end{array}$ & Compositae & Conyza & $\begin{array}{l}\text { Conyza } \\
\text { canadensis }\end{array}$ & $\begin{array}{l}\text { Perennial } \\
\text { herb }\end{array}$ \\
\hline $\begin{array}{l}\text { Lapsana } \\
\text { apogonoide } \\
\text {-s }\end{array}$ & Compositae & $\begin{array}{l}\text { Lapsanastr } \\
\text { um }\end{array}$ & $\begin{array}{l}\text { Lapsanastrum } \\
\text { apogonoides }\end{array}$ & $\begin{array}{l}\text { Biennial } \\
\text { herb }\end{array}$ \\
\hline Sowthistle & Compositae & Sonchus & $\begin{array}{l}\text { Sonchus } \\
\text { oleraceus }\end{array}$ & $\begin{array}{l}\text { Annual } \\
\text { herb }\end{array}$ \\
\hline Dandelion & Compositae & Taraxacum & $\begin{array}{l}\text { Taraxacum } \\
\text { mongolicum }\end{array}$ & $\begin{array}{l}\text { Perennial } \\
\text { herb }\end{array}$ \\
\hline $\begin{array}{l}\text { Asiatic } \\
\text { plantain } \\
\text { herb }\end{array}$ & Plantaginaceae & Plantago & $\begin{array}{l}\text { Plantago } \\
\text { asiatica }\end{array}$ & $\begin{array}{l}\text { Perennial } \\
\text { herb }\end{array}$ \\
\hline $\begin{array}{l}\text { Chinese } \\
\text { lobelia herb }\end{array}$ & Chinese lobelia & Lobelia & $\begin{array}{l}\text { Lobelia } \\
\text { chinensis }\end{array}$ & $\begin{array}{l}\text { Perennial } \\
\text { herb }\end{array}$ \\
\hline $\begin{array}{l}\text { Pedunculat- } \\
\text { e trigonotis } \\
\text { herb }\end{array}$ & Boraginaceae & Trigonotis & $\begin{array}{l}\text { Trigonotis } \\
\text { peduncularis }\end{array}$ & $\begin{array}{l}\text { Biennial } \\
\text { herb }\end{array}$ \\
\hline $\begin{array}{l}\text { Black } \\
\text { nightshade } \\
\text { herb }\end{array}$ & Solanaceae & Solanum & $\begin{array}{l}\text { Solanum } \\
\text { nigrum }\end{array}$ & $\begin{array}{l}\text { Perennial } \\
\text { herb }\end{array}$ \\
\hline $\begin{array}{l}\text { Brittle } \\
\text { falsepimper } \\
\text { nel herb }\end{array}$ & $\begin{array}{l}\text { Scrophulariace } \\
\text { ae }\end{array}$ & Lindernia & $\begin{array}{l}\text { Lindernia } \\
\text { crustacea }\end{array}$ & $\begin{array}{l}\text { Annual } \\
\text { herb }\end{array}$ \\
\hline $\begin{array}{l}\text { Fortune } \\
\text { paolownia } \\
\text { flower }\end{array}$ & $\begin{array}{l}\text { Scrophulariace } \\
\text { ae }\end{array}$ & Paulownia & $\begin{array}{l}\text { Paulownia } \\
\text { fortunei }\end{array}$ & $\begin{array}{l}\text { Deciduous } \\
\text { tree }\end{array}$ \\
\hline $\begin{array}{l}\text { Creeping } \\
\text { rostellularia } \\
\text { herb }\end{array}$ & Acanthaceae & Justicia & $\begin{array}{l}\text { Justicia } \\
\text { procumbens }\end{array}$ & $\begin{array}{l}\text { Perennial } \\
\text { herb }\end{array}$ \\
\hline $\begin{array}{l}\text { Negundo } \\
\text { chastetree }\end{array}$ & Verbenaceae & Vitex & Vitex negundo & $\begin{array}{l}\text { Deciduous } \\
\text { shrub }\end{array}$ \\
\hline $\begin{array}{l}\text { Hempleaf } \\
\text { negundo } \\
\text { chastetree }\end{array}$ & Verbenaceae & Vitex & $\begin{array}{l}\text { Vitex negundo } \\
\text { var. } \\
\text { cannabifolia }\end{array}$ & $\begin{array}{l}\text { Deciduous } \\
\text { shrub }\end{array}$ \\
\hline $\begin{array}{l}\text { Slender } \\
\text { clinopodiu- } \\
\text { m herb }\end{array}$ & Labiatae & $\begin{array}{l}\text { Clinopodiu } \\
-m\end{array}$ & $\begin{array}{l}\text { Clinopodium } \\
\text { gracile }\end{array}$ & $\begin{array}{l}\text { Perennial } \\
\text { herb }\end{array}$ \\
\hline $\begin{array}{l}\text { Nodalflowe } \\
-r \\
\text { synedrella } \\
\text { herb }\end{array}$ & $\begin{array}{l}\text { Hydrocharitace } \\
\text { ae }\end{array}$ & Vallisneria & $\begin{array}{l}\text { Vallisneria } \\
\text { natans }\end{array}$ & $\begin{array}{l}\text { Perennial } \\
\text { submerged } \\
\text { aquatic } \\
\text { grass }\end{array}$ \\
\hline $\begin{array}{l}\text { Curly } \\
\text { pondweed }\end{array}$ & $\begin{array}{l}\text { Potamogetonac } \\
\text { eae }\end{array}$ & $\begin{array}{l}\text { Potamoget- } \\
\text { on }\end{array}$ & $\begin{array}{l}\text { Potamogeton } \\
\text { crispus }\end{array}$ & $\begin{array}{l}\text { Perennial } \\
\text { submerged } \\
\text { aquatic }\end{array}$ \\
\hline
\end{tabular}

\begin{tabular}{|c|c|c|c|c|}
\hline Plant name & Family & Genus & Latin name & Category \\
\hline & & & & grass \\
\hline $\begin{array}{l}\text { Common } \\
\text { dayflower } \\
\text { herb }\end{array}$ & $\begin{array}{l}\text { Commelinacea } \\
e\end{array}$ & Commelina & $\begin{array}{l}\text { Commelina } \\
\text { communis }\end{array}$ & $\begin{array}{l}\text { Annual } \\
\text { herb }\end{array}$ \\
\hline $\begin{array}{l}\text { Hupeh } \\
\text { liriope root } \\
\text { tuber }\end{array}$ & Liliaceae & Liriope & Liriope spicata & $\begin{array}{l}\text { Perennial } \\
\text { herb }\end{array}$ \\
\hline $\begin{array}{l}\text { Bodinier } \\
\text { lilyturf root } \\
\text { tuber }\end{array}$ & Liliaceae & $\begin{array}{l}\text { Ophiopogo } \\
-n\end{array}$ & $\begin{array}{l}\text { Ophiopogon } \\
\text { bodinieri }\end{array}$ & $\begin{array}{l}\text { Perennial } \\
\text { herb }\end{array}$ \\
\hline $\begin{array}{l}\text { Dwarf } \\
\text { lilyturf root }\end{array}$ & Liliaceae & $\begin{array}{l}\text { Ophiopogo } \\
-n\end{array}$ & $\begin{array}{l}\text { Ophiopogon } \\
\text { japonicus }\end{array}$ & $\begin{array}{l}\text { Perennial } \\
\text { herb }\end{array}$ \\
\hline $\begin{array}{l}\text { Taro } \\
\text { rhizome }\end{array}$ & Araceae & Colocasia & $\begin{array}{l}\text { Colocasia } \\
\text { esculentum var. } \\
\text { antiquorum }\end{array}$ & $\begin{array}{l}\text { Perennial } \\
\text { herb }\end{array}$ \\
\hline $\begin{array}{l}\text { Common } \\
\text { ducksmeat } \\
\text { herb }\end{array}$ & Lemnaceae & Lemna & Lemna minor & $\begin{array}{l}\text { Annual } \\
\text { floating } \\
\text { herb }\end{array}$ \\
\hline $\begin{array}{l}\text { Common } \\
\text { ducksmeat } \\
\text { herb }\end{array}$ & Lemnaceae & Spirodela & $\begin{array}{l}\text { Spirodela } \\
\text { polyrrhiza }\end{array}$ & $\begin{array}{l}\text { Perenial } \\
\text { floating } \\
\text { herb }\end{array}$ \\
\hline $\begin{array}{l}\text { Reed } \\
\text { canary- } \\
\text { grass }\end{array}$ & Cyperaceae & $\begin{array}{l}\text { Fimbristyli } \\
s\end{array}$ & $\begin{array}{l}\text { Fimbristylis } \\
\text { miliacea }\end{array}$ & $\begin{array}{l}\text { Perennial } \\
\text { herb }\end{array}$ \\
\hline $\begin{array}{l}\text { Shortleaf } \\
\text { kyllinga } \\
\text { herb }\end{array}$ & Cyperaceae & Kyllinga & $\begin{array}{l}\text { Kyllinga } \\
\text { brevifolia }\end{array}$ & $\begin{array}{l}\text { Perennial } \\
\text { herb }\end{array}$ \\
\hline $\begin{array}{l}\text { Prairie } \\
\text { junegrass }\end{array}$ & Gramineae & $\begin{array}{l}\text { Beckmanni- } \\
a\end{array}$ & $\begin{array}{l}\text { Beckmannia } \\
\text { syzigachne }\end{array}$ & $\begin{array}{l}\text { Perennial } \\
\text { herb }\end{array}$ \\
\hline $\begin{array}{l}\text { Common } \\
\text { crabgrass } \\
\text { herb }\end{array}$ & Gramineae & Digitaria & $\begin{array}{l}\text { Digitaria } \\
\text { ciliaris }\end{array}$ & $\begin{array}{l}\text { Annual } \\
\text { herb }\end{array}$ \\
\hline $\begin{array}{l}\text { Common } \\
\text { crabgrass }\end{array}$ & Gramineae & Digitaria & $\begin{array}{l}\text { Digitaria } \\
\text { sanguinalis }\end{array}$ & $\begin{array}{l}\text { Annual } \\
\text { herb }\end{array}$ \\
\hline $\begin{array}{l}\text { Miscanthus } \\
\text { floridulus }\end{array}$ & Gramineae & Miscanthus & $\begin{array}{l}\text { Miscanthus } \\
\text { floridulus }\end{array}$ & $\begin{array}{l}\text { Perennial } \\
\text { herb }\end{array}$ \\
\hline $\begin{array}{l}\text { Folium } \\
\text { bambosae }\end{array}$ & Gramineae & $\begin{array}{l}\text { Oplismenu- } \\
s\end{array}$ & $\begin{array}{l}\text { Oplismenus } \\
\text { compositus }\end{array}$ & $\begin{array}{l}\text { Perennial } \\
\text { herb }\end{array}$ \\
\hline Oplismenus & Gramineae & $\begin{array}{l}\text { Oplismenu- } \\
s\end{array}$ & $\begin{array}{l}\text { Oplismenus } \\
\text { undulatifolius }\end{array}$ & $\begin{array}{l}\text { Perennial } \\
\text { herb }\end{array}$ \\
\hline $\begin{array}{l}\text { Climbing } \\
\text { fern }\end{array}$ & Lygodiaceae & Lygodium & $\begin{array}{l}\text { Lygodium } \\
\text { japonicum }\end{array}$ & Fern \\
\hline $\begin{array}{l}\text { Serrulate } \\
\text { brake herb }\end{array}$ & Pteridaceae & Pteris & Pteris multifida & Fern \\
\hline
\end{tabular}

\section{Problems and suggestions}

\subsection{Types and proportion of indigenous plants in urban greening plants in Nanchang}

Indigenous plants account for $21.29 \%$ among all greening plants in Nanchang, where the gymnosperm takes up $66.67 \%$, and the angiosperm takes up $21.35 \%$. Nanchang has a wide spectrum of indigenous plants that can be used for greening purposes, but currently, a few indigenous plants like masson pines, cinnamomum camphora, osmanthus fragrans, and claeocarpus glabripetalus have been overused in urban greening, while lots of other indigenous plants are largely overlooked. Aside from several species like Cinnamomum camphora, white oak, sweetgum, and sasanqua that have been used in greening areas in Nanchang, many other indigenous plants including Cephalotaxus fortunei, Lindera aggregate, Schima superba, Castanopsis fargesii, Castanopsis sclerophylla, Cyclobalanopsis glauca, Quercus variabilis, Cyclobalanopsis jenseniana, Symplocos sumuntia, Ilex chinensis, and Sambucus williamsii are hardly used for greening purposes in the city.

\subsection{Utilization rate of indigenous plants for greening purposes in Nanchang}

The utilization rate of indigenous plants for greening purposes in Nanchang approaches $17 \%$, and the city still 
relies more on introduced plants for greening. Protecting species diversity is a premise of protecting biodiversity. Therefore, plant landscaping should rely mainly on indigenous plants and take introduced plants as supplements, and the proportion of indigenous trees in the overall number of trees for greening in the city should be above $80 \%$. Investigations also revealed that there are over 60 invasive species in Nanchang, and hence it is necessary to be cautious in introducing plants from outside to reduce the risk of invasion of species, especially the herbaceous plants that may cause ecological hazards.

\subsection{Proportion of different categories of indigenous plants in greening plants in Nanchang}

(1) Among greening plants in Nanchang, there are 16 species of evergreen indigenous trees and 25 species of deciduous indigenous trees. The local vegetation of Nanchang is evergreen broad-leaf forests, with evergreen trees being the dominating species. In urban greening, evergreen tree species take up a large proportion, and hence presents a pattern of "green throughout seasons". Thus, there are viewable sceneries throughout the year, and the sceneries are of distinct seasonal features. Therefore, evergreen tree species account for above $65 \%$ of the overall tree species used for greening in Nanchang.

(2) Among all species used for greening in Nanchang, there are 17 species of indigenous trees and 17 species of shrubs. Trees play a dominating role, have good ornamental effects, and can be used for shading of buildings, isolated planting, and shading along sidewalks. The shrubs, though playing a less dominating role, are of high ornamental value and can enrich the diversity of sceneries. They can be used as green fences, quickset hedges, or ground covers, and can be trimmed into different shapes for ornamental purposes. Besides, they are often co-planted with trees to create multi-layered community structures of trees, shrubs, and grasses. Thus, the optimal ratio of trees to shrubs in greening should be $1: 3$.

\subsection{Vertical greening}

Among all species used for greening purposes in Nanchang, there are 7 species of indigenous vines (2 species of evergreen vines, and 5 species of deciduous vines), taking up $17.5 \%$ of all indigenous vines in the region, a very small proportion. Indigenous vines of great potentials include Stauntonia obovatifoliola, Aristolochia debilis, Actinidia chinensis, Clematis finetiana, and Akebia quinate, the flowers, fruits and gestures of which are of high ornamental value, and these species have strong adaptability, feature easy maintenance and good ecological effects.

\section{Conclusion}

Biodiversity in cities is based on diversity of landscape plants. Excessive introduction of species from outside will inevitably result in gradual disappearance of indigenous plants, reduce species diversity, undermine the local specialty of landscapes, and thereby reduce biodiversity. Nanchang boasts a rich reserve of indigenous plants, but the utilization rate of these plants remains low, and introduced plants play a dominating role in greening; the matching of evergreen and deciduous plants, the trees, shrubs and vines is disproportionate. Investigation of the resources of indigenous plants in Nanchang in this study is expected to provide more options for greening in the city, reduce the risk of species invasion, increase diversity of plants in the region, promote better development of indigenous plants in Nanchang and lead Nanchang towards the goal of becoming a "garden city".

\section{Acknowledgements}

This paper is a phased research result of "Research on the suitability index of indigenous trees in urban landscape in Nanchang", a scientific and technological research project funded by Department of Education of Jiangxi (GJJ161107).

\section{References}

1. Li S.H. (2005). Constructing greening landscapes dominated by indigenous plants. Chinese Landscape Architecture, 1:47.

2. Lin Y. (1993). Flora of Jiangxi (Vol. I). Jiangxi Science and Technology Press, Nanchang.

3. Editorial board of Flora of Jiangxi (2004). Flora of Jiangxi (Vol. II). Jiangxi Science and Technology Press, Nanchang.

4. Editorial board of Flora of Jiangxi (2014). Flora of Jiangxi (Vol. III). Jiangxi Science and Technology Press, Nanchang. 